\title{
The algebra of polynomial integro-differential operators is a holonomic bimodule over the subalgebra of polynomial differential operators
}

\author{
V. V. Bavula
}

\begin{abstract}
In contrast to its subalgebra $A_{n}:=K\left\langle x_{1}, \ldots, x_{n}, \frac{\partial}{\partial x_{1}}, \ldots, \frac{\partial}{\partial x_{n}}\right\rangle$ of polynomial differential operators (i.e. the $n$ 'th Weyl algebra), the algebra $\mathbb{I}_{n}:=K\left\langle x_{1}, \ldots, x_{n}, \frac{\partial}{\partial x_{1}}, \ldots, \frac{\partial}{\partial x_{n}}, \int_{1}, \ldots, \int_{n}\right\rangle$ of polynomial integro-differential operators is neither left nor right Noetherian algebra; moreover it contains infinite direct sums of nonzero left and right ideals. It is proved that $\mathbb{I}_{n}$ is a left (right) coherent algebra iff $n=1$; the algebra $\mathbb{I}_{n}$ is a holonomic $A_{n}$-bimodule of length $3^{n}$ and has multiplicity $3^{n}$, and all $3^{n}$ simple factors of $\mathbb{I}_{n}$ are pairwise non-isomorphic $A_{n}$-bimodules. The socle length of the $A_{n}$-bimodule $\mathbb{I}_{n}$ is $n+1$, the socle filtration is found, and the $m$ 'th term of the socle filtration has length $\left(\begin{array}{c}n \\ m\end{array}\right) 2^{n-m}$. This fact gives a new canonical form for each polynomial integro-differential operator. It is proved that the algebra $\mathbb{I}_{n}$ is the maximal left (resp. right) order in the largest left (resp. right) quotient ring of the algebra $\mathbb{I}_{n}$.

Key Words: the algebra of polynomial integro-differential operators, the Weyl algebra, the socle, the socle length.

Mathematics subject classification 2000: 16D60, 16S32.
\end{abstract}

\section{Introduction}

Throughout, ring means an associative ring with $1 ;$ module means a left module; $\mathbb{N}:=\{0,1, \ldots\}$ is the set of natural numbers; $K$ is a field of characteristic zero and $K^{*}$ is its group of units; $P_{n}:=K\left[x_{1}, \ldots, x_{n}\right]$ is a polynomial algebra over $K ; \partial_{1}:=\frac{\partial}{\partial x_{1}}, \ldots, \partial_{n}:=\frac{\partial}{\partial x_{n}}$ are the partial derivatives ( $K$-linear derivations) of $P_{n} ; \operatorname{End}_{K}\left(P_{n}\right)$ is the algebra of all $K$-linear maps from $P_{n}$ to $P_{n}$; the subalgebras $A_{n}:=K\left\langle x_{1}, \ldots, x_{n}, \partial_{1}, \ldots, \partial_{n}\right\rangle$ and $\mathbb{I}_{n}:=K\left\langle x_{1}, \ldots, x_{n}, \partial_{1}, \ldots, \partial_{n}, \int_{1}, \ldots, \int_{n}\right\rangle$ of the algebra $\operatorname{End}_{K}\left(P_{n}\right)$ are called the $n$ 'th Weyl algebra and the algebra of polynomial integrodifferential operators respectively.

The Weyl algebras $A_{n}$ are Noetherian algebras and domains. The algebras $\mathbb{I}_{n}$ are neither left nor right Noetherian and not domains. Moreover, they contain infinite direct sums of nonzero left and right ideals [1]. The algebra $A_{n}$ is isomorphic to its opposite algebra $A_{n}^{o p}$ via the $K$-algebra involution:

$$
A_{n} \rightarrow A_{n}, \quad x_{i} \mapsto \partial_{i}, \quad \partial_{i} \mapsto x_{i}, \quad i=1, \ldots, n .
$$

Therefore, every $A_{n}$-bimodule is a left $A_{2 n}$-module and vice versa. Inequality of Bernstein [5] states that each nonzero finitely generated $A_{n}$-module has Gelfand-Kirillov dimension which is greater or equal to $n$. A finitely generated $A_{n}$-module is holonomic if it has Gelfand-Kirillov dimension $n$. The holonomic $A_{n}$-modules share many pleasant properties. In particular, all holonomic modules have finite length, each nonzero submodule and factor module of a holonomic module is holonomic. The aim of the paper is to prove Theorem 2.5. In particular, to show that the algebra $\mathbb{I}_{n}$ is a holonomic $A_{n}$-bimodule of length $3^{n}$ and has multiplicity $3^{n}$, i.e. a holonomic left $A_{2 n}$-module of length $3^{n}$ and has multiplicity $3^{n}$. All $3^{n}$ simple factors of $\mathbb{I}_{n}$ are pairwise non-isomorphic $A_{n}$-bimodules. We also found the socle filtration of the $A_{2 n}$-module $\mathbb{I}_{n}$. It turns out that the socle length of the $A_{2 n}$-module is $n+1$, and the length, as an $A_{2 n}$-module, of the $m$ 'th socle factor is $\left(\begin{array}{c}n \\ m\end{array}\right) 2^{n-m}$ (Theorem 2.5. (4)) where $m=0,1, \ldots, n$. A new $K$-basis for the algebra $\mathbb{I}_{n}$ is found which gives a new canonical form for each polynomial integro-differential operator, see (16). By the 
very definition, $\mathbb{I}_{n}=\bigotimes_{i=1}^{n} \mathbb{I}_{1}(i) \simeq \mathbb{I}_{1}^{\otimes n}$ where $\mathbb{I}_{1}(i):=K\left\langle x_{i}, \partial_{i}, \int_{i}\right\rangle$ and $A_{n}=\bigotimes_{i=1}^{n} A_{1}(i)=A_{1}^{\otimes n}$ where $A_{1}(i):=K\left\langle x_{i}, \partial_{i}\right\rangle$. So, the properties of the algebras $\mathbb{I}_{n}$ and $A_{n}$ are 'determined' by the properties of the algebras $\mathbb{I}_{1}$ and $A_{1}$.

At the beginning of Section 2 we collect necessary facts on the algebras $\mathbb{I}_{n}$. Then we prove Theorem 2.5 in the case when $n=1$ and prove some necessary results that are used in the proof of Theorem 2.5 (in the general case) which is given at the end of the section.

In Section 3, it is proved that the algebra $\mathbb{I}_{n}$ is left (right) coherent iff $n=1$ (Theorem 3.1).

In Section 4 , it is proved that the algebra $\mathbb{I}_{n}$ is the maximal left (resp. right) order in its largest left (resp. right) quotient ring (Theorem 4.3).

\section{Proof of Theorem 2.5}

At the beginning of this section, we collect necessary (mostly elementary) facts on the algebra $\mathbb{I}_{1}$ from [1] that are used later in the paper.

The algebra $\mathbb{I}_{1}$ is generated by the elements $\partial, H:=\partial x$ and $\int$ (since $x=\int H$ ) that satisfy the defining relations (Proposition 2.2, 1]):

$$
\partial \int=1,\left[H, \int\right]=\int,[H, \partial]=-\partial, H\left(1-\int \partial\right)=\left(1-\int \partial\right) H=1-\int \partial,
$$

where $[a, b]:=a b-b a$ is the commutator of elements $a$ and $b$. The elements of the algebra $\mathbb{I}_{1}$,

$$
e_{i j}:=\int^{i} \partial^{j}-\int^{i+1} \partial^{j+1}, \quad i, j \in \mathbb{N},
$$

satisfy the relations $e_{i j} e_{k l}=\delta_{j k} e_{i l}$ where $\delta_{j k}$ is the Kronecker delta function and $\mathbb{N}:=\{0,1, \ldots\}$ is the set of natural numbers. Notice that $e_{i j}=\int^{i} e_{00} \partial^{j}$. The matrices of the linear maps $e_{i j} \in \operatorname{End}_{K}(K[x])$ with respect to the basis $\left\{x^{[s]}:=\frac{x^{s}}{s !}\right\}_{s \in \mathbb{N}}$ of the polynomial algebra $K[x]$ are the elementary matrices, i.e.

$$
e_{i j} * x^{[s]}= \begin{cases}x^{[i]} & \text { if } j=s \\ 0 & \text { if } j \neq s .\end{cases}
$$

Let $E_{i j} \in \operatorname{End}_{K}(K[x])$ be the usual matrix units, i.e. $E_{i j} * x^{s}=\delta_{j s} x^{i}$ for all $i, j, s \in \mathbb{N}$. Then

$$
e_{i j}=\frac{j !}{i !} E_{i j}
$$

$K e_{i j}=K E_{i j}$, and $F:=\bigoplus_{i, j \geq 0} K e_{i j}=\bigoplus_{i, j \geq 0} K E_{i j} \simeq M_{\infty}(K)$, the algebra (without 1) of infinite dimensional matrices. $F$ is the only proper ideal (i.e. $\left.\neq 0, \mathbb{I}_{1}\right)$ of the algebra $\mathbb{I}_{1}[1]$.

$\mathbb{Z}$-grading on the algebra $\mathbb{I}_{1}$ and the canonical form of an integro-differential operator [1], [2]. The algebra $\mathbb{I}_{1}=\bigoplus_{i \in \mathbb{Z}} \mathbb{I}_{1, i}$ is a $\mathbb{Z}$-graded algebra $\left(\mathbb{I}_{1, i} \mathbb{I}_{1, j} \subseteq \mathbb{I}_{1, i+j}\right.$ for all $\left.i, j \in \mathbb{Z}\right)$ where

$$
\mathbb{I}_{1, i}= \begin{cases}D_{1} \int^{i}=\int^{i} D_{1} & \text { if } i>0, \\ D_{1} & \text { if } i=0, \\ \partial^{|i|} D_{1}=D_{1} \partial^{|i|} & \text { if } i<0,\end{cases}
$$

the algebra $D_{1}:=K[H] \bigoplus \bigoplus_{i \in \mathbb{N}} K e_{i i}$ is a commutative non-Noetherian subalgebra of $\mathbb{I}_{1}, H e_{i i}=$ $e_{i i} H=(i+1) e_{i i}$ for $i \in \mathbb{N}$ (and so $\bigoplus_{i \in \mathbb{N}} K e_{i i}$ is the direct sum of non-zero ideals $K e_{i i}$ of the algebra $\left.D_{1}\right) ;\left(\int^{i} D_{1}\right)_{D_{1}} \simeq D_{1}, \int^{i} d \mapsto d ; D_{1}\left(D_{1} \partial^{i}\right) \simeq D_{1}, d \partial^{i} \mapsto d$, for all $i \geq 0$ since $\partial^{i} \int^{i}=1$. Notice that the maps $\cdot \int^{i}: D_{1} \rightarrow D_{1} \int^{i}, d \mapsto d \int^{i}$, and $\partial^{i} \cdot: D_{1} \rightarrow \partial^{i} D_{1}, d \mapsto \partial^{i} d$, have the same kernel $\bigoplus_{j=0}^{i-1} K e_{j j}$.

Each element $a$ of the algebra $\mathbb{I}_{1}$ is the unique finite sum

$$
a=\sum_{i>0} a_{-i} \partial^{i}+a_{0}+\sum_{i>0} \int^{i} a_{i}+\sum_{i, j \in \mathbb{N}} \lambda_{i j} e_{i j}
$$


where $a_{k} \in K[H]$ and $\lambda_{i j} \in K$. This is the canonical form of the polynomial integro-differential operator [1].

Definition. Let $a \in \mathbb{I}_{1}$ be as in (41) and let $a_{F}:=\sum \lambda_{i j} e_{i j}$. Suppose that $a_{F} \neq 0$ then

$$
\operatorname{deg}_{F}(a):=\min \left\{n \in \mathbb{N} \mid a_{F} \in \bigoplus_{i, j=0}^{n} K e_{i j}\right\}
$$

is called the $F$-degree of the element $a ; \operatorname{deg}_{F}(0):=-1$.

sum

Let $v_{i}:=\left\{\begin{array}{ll}\int^{i} & \text { if } i>0, \\ 1 & \text { if } i=0, \\ \partial^{|i|} & \text { if } i<0 .\end{array}\right.$ Then $\mathbb{I}_{1, i}=D_{1} v_{i}=v_{i} D_{1}$ and an element $a \in \mathbb{I}_{1}$ is the unique finite

$$
a=\sum_{i \in \mathbb{Z}} b_{i} v_{i}+\sum_{i, j \in \mathbb{N}} \lambda_{i j} e_{i j}
$$

where $b_{i} \in K[H]$ and $\lambda_{i j} \in K$. So, the set $\left\{H^{j} \partial^{i}, H^{j}, \int{ }^{i} H^{j}, e_{s t} \mid i \geq 1 ; j, s, t \geq 0\right\}$ is a $K$-basis for the algebra $\mathbb{I}_{1}$. The multiplication in the algebra $\mathbb{I}_{1}$ is given by the rule:

$$
\begin{gathered}
\int H=(H-1) \int, H \partial=\partial(H-1), \int e_{i j}=e_{i+1, j}, \quad e_{i j} \int=e_{i, j-1}, \partial e_{i j}=e_{i-1, j} e_{i j} \partial=\partial e_{i, j+1} . \\
H e_{i i}=e_{i i} H=(i+1) e_{i i}, \quad i \in \mathbb{N},
\end{gathered}
$$

where $e_{-1, j}:=0$ and $e_{i,-1}:=0$.

The factor algebra $B_{1}:=\mathbb{I}_{1} / F$ is the simple Laurent skew polynomial algebra $K[H]\left[\partial, \partial^{-1} ; \tau\right]$ where the automorphism $\tau \in \operatorname{Aut}_{K-\operatorname{alg}}(K[H])$ is defined by the rule $\tau(H)=H+1$, [1]. Let

$$
\pi: \mathbb{I}_{1} \rightarrow B_{1}, \quad a \mapsto \bar{a}: a+F,
$$

be the canonical epimorphism.

The Weyl algebra $A_{2}$ is equipped with the, so-called, filtration of Bernstein, $A_{2}=\bigcup_{i \geq 0} A_{2, \leq i}$ where $A_{2, \leq i}:=\bigoplus\left\{K x_{1}^{\alpha_{1}} x_{2}^{\alpha_{2}} \partial_{1}^{\beta_{1}} \partial_{2}^{\beta_{2}} \mid \alpha_{1}+\alpha_{2}+\beta_{1}+\beta_{2} \leq i\right\}$. The polynomial algebra $P_{2}:=$ $K\left[x_{1}, x_{2}\right] \simeq A_{2} /\left(A_{2} \partial_{1}+A_{2} \partial_{2}\right)$ is a simple left $A_{2}$-module with $\operatorname{End}_{A_{2}}\left(P_{2}\right)=\operatorname{ker}_{P_{2}}\left(\partial_{1}\right) \cap \operatorname{ker}_{P_{2}}\left(\partial_{2}\right)=$ $K$. The standard filtration $\left\{A_{2, \leq i} \cdot 1\right\}_{i \in \mathbb{N}}$ of the $A_{2}$-module $P_{2}$ coincides with the filtration $\left\{P_{2, \leq i}:=\right.$ $\sum_{\alpha_{1}, \alpha_{2} \geq 0}\left\{K x_{1}^{\alpha_{1}} x_{2}^{\alpha_{2}} \mid \alpha_{1}+\alpha_{2} \leq i\right\}_{i \in \mathbb{N}}$ on the polynomial algebra $P_{2}$ by the total degree, i.e. $P_{2, \leq i}=$ $A_{2, \leq i} \cdot 1$ for all $i \geq 0$, and so $\operatorname{dim}_{K}\left(A_{2, \leq i}\right)=\frac{(i+1)(i+2)}{2}$. Therefore, $P_{2}$ is a holonomic $A_{2}$-module with multiplicity $e\left(P_{2}\right)=1$ and $\operatorname{End}_{A_{2}}\left(P_{2}\right) \simeq K$. The Weyl algebra $A_{1}$ admits the $K$-isomorphism:

$$
\xi: A_{1}, \rightarrow A_{1}, \quad x \mapsto \partial, \partial \mapsto-x .
$$

Then $1 \otimes \xi$ is an automorphism of the Weyl algebra $A_{2}$. The twisted by the automorphism $1 \otimes \xi$ $A_{2}$-module $P_{2}$,

$$
P_{2}^{1 \otimes \xi} \simeq K\left[x_{1}, \partial_{2}\right] \simeq A_{2} /\left(A_{2} \partial_{1}+A_{2} x_{2}\right)
$$

is a simple holonomic $A_{2}$-module with multiplicity 1 and $\operatorname{End}_{A_{2}}\left(P_{2}^{1 \otimes \xi}\right) \simeq K$.

The Weyl algebra $A_{1}$ is isomorphic to its opposite algebra $A_{1}^{o p}$ via

$$
A_{1} \rightarrow A_{1}^{o p}, \quad x \mapsto \partial, \partial \mapsto x
$$

In particular, each $A_{1}$-bimodule ${ }_{A_{1}} M_{A_{1}}$ is a left $A_{2}$-module: ${ }_{A_{1}} M_{A_{1}}={ }_{A_{1} \otimes A_{1}^{o p}} M \simeq{ }_{A_{1} \otimes A_{1}} M=$ $A_{2} M$.

Lemma 2.1 1. ${ }_{A_{1}} F_{A_{1}}=A_{1} e_{00} A_{1} \simeq{ }_{A_{1}}\left(A_{1} / A_{1} \partial \otimes A_{1} / x A_{1}\right)_{A_{1}}$. 
2. $A_{2} F \simeq A_{2} /\left(A_{2} \partial_{1}+A_{2} \partial_{2}\right) \simeq K\left[x_{1}, x_{2}\right]$ is a simple holonomic $A_{2}$-module with multiplicity 1 with respect to the filtration of Bernstein of the algebra $A_{2}$ and $\operatorname{End}_{A_{2}}(F) \simeq K$.

Proof. $A_{1}\left(A_{1} / A_{1} \partial \otimes A_{1} / x A_{1}\right)_{A_{1}} \simeq A_{1} \otimes A_{1}\left(A_{1} / A_{1} \partial \otimes A_{1} / A_{1} \partial\right) \simeq A_{2} /\left(A_{2} \partial_{1}+A_{2} \partial_{2}\right) \simeq K\left[x_{1}, x_{2}\right]$ is a simple holonomic $A_{2}$-module with multiplicity 1 with respect to the filtration of Bernstein of the algebra $A_{2}$ and $\operatorname{End}_{A_{2}}(F) \simeq K$. Clearly, ${ }_{A_{1}} F_{A_{1}}=A_{1} e_{00} A_{1}$ and the $A_{1}$-bimodule homomorphism

$$
A_{1} / A_{1} \partial \otimes A_{1} / x A_{1} \rightarrow A_{1} e_{00} A_{1}, \quad\left(1+A_{1} \partial_{1}\right) \otimes\left(1+x A_{1}\right) \mapsto e_{00},
$$

is an epimorphism. Therefore, it is an isomorphism by the simplicity of the first $A_{1}$-bimodule.

Proposition 2.2 1. $A_{1}\left(\mathbb{I}_{1} /\left(A_{1}+F\right)\right)_{A_{1}} \simeq A_{1} / A_{1} \partial \otimes A_{1} / \partial A_{1}$.

2. $A_{2}\left(\mathbb{I}_{1} /\left(A_{1}+F\right)\right) \simeq A_{1} / A_{1} \partial \otimes A_{1} / A_{1} x \simeq A_{2} /\left(A_{2} \partial_{1}+A_{2} x_{2}\right) \simeq K\left[x_{1}, \partial_{2}\right]$ is a simple holonomic $A_{2}$-module with multiplicity 1 with respect to the filtration of Bernstein and $\operatorname{End}_{A_{2}}\left(K\left[x_{1}, \partial_{2}\right]\right) \simeq$ $K$.

3. $A_{1}\left(\mathbb{I}_{1} /\left(A_{1}+F\right)\right) \simeq\left(A_{1} / A_{1} \partial\right)^{(\mathbb{N})} \simeq K[x]^{(\mathbb{N})}$ is a semi-simple left $A_{1}$-module and $\left(\mathbb{I}_{1} /\left(A_{1}+\right.\right.$ $F))_{A_{1}} \simeq\left(A_{1} / \partial A_{1}\right)^{(\mathbb{N})} \simeq K[x]^{(\mathbb{N})}$ is a semi-simple right $A_{1}$-module.

Proof. 1 and 2. Notice that $A_{2}\left(A_{1} / A_{1} \partial \otimes A_{1} / A_{1} x\right) \simeq A_{A_{2}}\left(A_{2} /\left(A_{2} \partial_{1}+A_{2} x_{2}\right)\right) \simeq K\left[x_{1}, \partial_{2}\right]$ is a simple holonomic $A_{2}$-module with multiplicity 1 with respect to the filtration of Bernstein and $\operatorname{End}_{A_{2}}\left(K\left[x_{1}, \partial_{2}\right]\right) \simeq K$. The natural filtration of the polynomial algebra $Q^{\prime}:=K\left[x_{1}, \partial_{2}\right]=$ $\bigcup_{i \geq 0} Q_{\leq i}^{\prime}$ by the total degree of the variables, i.e. $Q_{\leq i}^{\prime}:=\bigoplus_{s+t \leq i} K x_{1}^{s} \partial_{2}^{t}$, is a standard filtration for the $A_{2}$-module $Q^{\prime}=A_{2} \cdot 1$ since $Q_{\leq i}^{\prime}=A_{2, \leq i} \cdot 1$ for all $i \geq 0$. In particular, $\operatorname{dim}_{K}\left(Q_{\leq i}^{\prime}\right)=\frac{(i+1)(i+2)}{2}$ for all $i \geq 0$. By (44), the $A_{1}$-bimodule $Q:=\mathbb{I}_{1} /\left(A_{1}+F\right)$ is the direct sum

$$
Q=\bigoplus_{i \geq 1} Q_{i}
$$

of its vector subspaces

$\left(Q_{i}\right)_{K[H]} \simeq \int^{i} K[H] / x^{i} K[H] \simeq \int^{i} K[H] / \int^{i}(H(H+1) \cdots(H+i-1)) \simeq K[H] /(H(H+1) \cdots(H+i-1))$

(since $x^{i}=\left(\int H\right)^{i}=\int^{i} H(H+1) \cdots(H+i-1)$ and $\left.\partial^{i} \int^{i}=1\right)$ such that $x Q_{i} \subseteq Q_{i+1}, Q_{i} x \subseteq Q_{i+1}$, $\partial Q_{i} \subseteq Q_{i-1}$ and $Q_{i} \partial \subseteq Q_{i-1}$ for all all $i \geq 1$ where $Q_{0}:=0$. Then $A_{2}$-module $Q$ has the finite dimensional ascending filtration $Q=\bigcup_{i \geq 0} Q_{\leq i}$ where $Q_{\leq i}:=\bigoplus_{1 \leq j \leq i+1} Q_{j}$ and

$$
\operatorname{dim}_{K}\left(Q_{\leq i}\right)=\sum_{j=0}^{i}(j+1)=\frac{(i+1)(i+2)}{2} \text { for all } i \geq 0 .
$$

Since $\partial Q_{1}=Q_{1} \partial=0$, the simple filtered $A_{2}$-module (treated as $A_{1}$-bimodule) ${ }_{A_{1}} Q_{A_{1}}^{\prime}=A_{1} / A_{1} \partial \otimes$ $A_{1} / \partial A_{1}$ can be seen as a filtered $A_{2}$-submodule of $Q$ via $\left(1+A_{1} \partial\right) \otimes\left(1+\partial A_{1}\right) \mapsto \int+A_{1}+F$. In particular, for all $i \geq 0$, we have the inclusions $Q_{i}^{\prime} \subseteq Q_{i}$ which are, in fact, equalities since $\operatorname{dim}_{K}\left(Q_{i}^{\prime}\right)=\operatorname{dim}_{K}\left(Q_{i}\right)$. Then,

$$
{ }_{A_{1}}\left(\mathbb{I}_{1} /\left(A_{1}+F\right)\right)_{A_{1}} \simeq{ }_{A_{1}}\left(A_{1} / A_{1} \partial \otimes A_{1} / \partial A_{1}\right)_{A_{1}} \simeq A_{2}\left(A_{1} / A_{1} \partial \otimes A_{1} / A_{1} x\right) \simeq K\left[x_{1}, \partial_{2}\right] .
$$

It is obvious that the $A_{2}$-module $K\left[x_{1}, \partial_{2}\right]$ is a simple $A_{2}$-module with multiplicity 1 and $\operatorname{End}_{A_{2}}\left(K\left[x_{1}, \partial_{2}\right]\right)$ $\simeq K$.

3. Statement 3 follows from statement 1 .

A linear map $\varphi$ acting in a vector space $V$ is called a locally nilpotent map if $V=\bigcup_{i \geq 1} \operatorname{ker}\left(\varphi^{i}\right)$, i.e. for each element $v \in V$ there exists a natural number $i$ such that $\varphi^{i}(v)=0$. 
It follows from Proposition 2.2 and (12) that

$$
\operatorname{ker}_{\mathbb{I}_{1} /\left(A_{1}+F\right)}(\partial \cdot) \bigcap \operatorname{ker}_{\mathbb{I}_{1} /\left(A_{1}+F\right)}(\cdot \partial)=K\left(\int+A_{1}+F\right),
$$

and that the maps $\partial \cdot: \mathbb{I}_{1} /\left(A_{1}+F\right) \rightarrow \mathbb{I}_{1} /\left(A_{1}+F\right), u \mapsto \partial u$, and $\cdot \partial: \mathbb{I}_{1} /\left(A_{1}+F\right) \rightarrow \mathbb{I}_{1} /\left(A_{1}+F\right)$, $u \mapsto u \partial$, are locally nilpotent since

$$
\partial * x_{1}^{i} x_{2}^{j}=i x_{1}^{i-1} x_{2}^{j}, \quad x_{1}^{i} x_{2}^{j} * \partial=-j x_{1}^{i} x_{2}^{j-1} .
$$

Recall that the $\operatorname{socle} \operatorname{soc}_{A}(M)$ of a module $M$ over a ring $A$ is the sum of all the simple submodules of $M$, if they exist, and zero, otherwise.

Theorem 2.3 1. The $A_{1}$-bimodule $\mathbb{I}_{1}$ is a holonomic $A_{2}$-module of length 3 with simple nonisomorphic factors $F \simeq A_{2} K\left[x_{1}, x_{2}\right], A_{1} A_{1} A_{1}$ and ${ }_{A_{2}} K\left[x_{1}, \partial_{2}\right]$. Each factor is a simple holonomic $A_{2}$-module with multiplicity 1 and its $A_{2}$-module endomorphism algebra is $K$.

2. $\operatorname{soc}_{A_{2}}\left(\mathbb{I}_{1}\right)=A_{1} \oplus F$.

3. The short exact sequence of $A_{2}$-modules

$$
0 \rightarrow A_{1} \bigoplus F \rightarrow \mathbb{I}_{1} \rightarrow \mathbb{I}_{1} /\left(A_{1}+F\right) \rightarrow 0
$$

is non-split.

Proof. 1. Statement 1 follows from Lemma 2.1, Proposition 2.2 and (15).

3 . Suppose that the short exact sequence of $A_{1}$-bimodules splits, we seek a contradiction. Then, by Proposition 2.2 (1) and (13), there is a nonzero element, say $u=\int+a+f \in \mathbb{I}_{1}$ with $a \in A_{1}$ and $f \in F$ such that $\partial u=0$ and $u \partial=0$. The first equation implies $1+\partial a=-\partial f \in A_{1} \cap F=0$, and so $\partial a=-1$ in $A_{1}$, a contradiction.

2. Statement 2 follows from statement 3 .

New basis for the algebra $\mathbb{I}_{n}$. It follows from (11), (12) and (15) that

$$
\mathbb{I}_{1}=\bigoplus_{i, j \geq 0} K x^{i} \partial^{j} \oplus \bigoplus_{k, l \geq 0} K e_{k l} \oplus \bigoplus\left\{K \int^{s} H^{t} \mid s \geq 1, t=0,1, \ldots, s-1\right\} .
$$

This gives a new $K$-basis for the algebra $\mathbb{I}_{1}:\left\{x^{i} \partial^{j}, e_{k l}, \int^{s} H^{t} \mid i, j, k, l \geq 0 ; s \geq 1 ; t=0,1, \ldots, s-1\right\}$. By taking $n$ 'th tensor product of this basis we obtain a new $K$-basis for the algebra $\mathbb{I}_{n}=\mathbb{I}_{1}^{\otimes n}$.

Lemma 2.4 1. The $A_{1}$-bimodule $\mathbb{I}_{1} / A_{1}$ is a holonomic $A_{2}$-module of length 2 with simple nonisomorphic factors $F \simeq{ }_{A_{2}} K\left[x_{1}, x_{2}\right]$ and ${ }_{A_{2}} K\left[x_{1}, \partial_{2}\right]$. Each factor is a simple holonomic $A_{2}$-module with multiplicity 1 and its $A_{2}$-module endomorphism algebra is $K$.

2. $\operatorname{soc}_{A_{2}}\left(\mathbb{I}_{1} / A_{1}\right)=F$.

3. The short exact sequence of $A_{2}$-modules

$$
0 \rightarrow F \rightarrow \mathbb{I}_{1} / A_{1} \rightarrow \mathbb{I}_{1} /\left(A_{1}+F\right) \rightarrow 0
$$

is non-split.

4. The short exact sequence of left $A_{1}$-modules 17$)$ splits and so $A_{1}\left(\mathbb{I}_{1} / A_{1}\right) \simeq K[x]^{(\mathbb{N})}$ is a semi-simple left $A_{1}$-module.

5. The short exact sequence of right $A_{1}$-modules (17) does not split, and so $\left(\mathbb{I}_{1} / A_{1}\right)_{A_{1}}$ is not a semi-simple right $A_{1}$-module. 
Proof. 1. Statement 1 follows from Theorem 2.3.(1).

3. Suppose that the short exact sequence of $A_{1}$-bimodules (17) splits, we seek a contradiction. Then, by Proposition 2.2. (1) and (13), there is a nonzero element, say $u=\int+f+A_{1} \in \mathbb{I}_{1} / A_{1}$ with $f \in F$ such that $0=\partial u=1+\partial f$ and $0=u \partial=1-e_{00}+f \partial$ in $\mathbb{I}_{1} / A_{1}$. The first equality gives $\partial f=0$ in $\mathbb{I}_{1} / A_{1}$, and so $f=\sum_{i \geq 0} \lambda_{i} e_{0 i}$ for some $\lambda_{i} \in K$. Then the second equality gives $e_{00}=f \partial=\sum_{i \geq 0} \lambda_{i} e_{0 i} \partial=\sum_{i \geq 0} \lambda_{i} e_{0, i+1}$, a contradiction.

2. Statement 2 follows from statement 3 .

4. Let $L$ be the last sum in the decomposition (16), i.e.

$$
\mathbb{I}_{1}=A_{1} \bigoplus F \bigoplus L
$$

Then $A_{1} \oplus L$ is a left $A_{1}$-submodule of ${ }_{A_{1}} \mathbb{I}_{1}$ since $\partial \int=1, x=\int H$ and $\int H=(H-1) \int$. Notice that $A_{1} \bigoplus L$ is not a right $A_{1}$-submodule of $\mathbb{I}_{1}$ since $\int \partial=1-e_{00} \notin A_{1} \oplus L$. By (18), $A_{1}\left(\mathbb{I}_{1} / A_{1}\right) \simeq F \bigoplus\left(A_{1}+L\right) / A_{1}$ is a direct sum of left $A_{1}$-submodules such that $A_{1} F \simeq K[x]^{(\mathbb{N})}$ (Lemma 2.1] (1)) and $A_{1}\left(\left(A_{1}+L\right) / A_{1}\right) \simeq \mathbb{I}_{1} /\left(A_{1}+F\right) \simeq K[x]^{(\mathbb{N})}$ (Proposition 2.2. (3)). Therefore, $A_{1}\left(\mathbb{I}_{1} / A_{1}\right)$ is a semi-simple module. Therefore, the short exact sequence of left $A_{1}$-modules (17) splits and $A_{1}\left(\mathbb{I}_{1} / A_{1}\right) \simeq K[x]^{(\mathbb{N})} \bigoplus K[x]^{(\mathbb{N})} \simeq K[x]^{(\mathbb{N})}$.

5. By Proposition 2.2. (1), $\left(\mathbb{I}_{1} /\left(A_{1}+F\right)\right)_{A_{1}} \simeq\left(A_{1} / \partial A_{1}\right)^{(\mathbb{N})}$. Suppose that the short exact sequence of right $A_{1}$-modules (17) splits, we seek a contradiction. In the factor module $\mathbb{I}_{1} /\left(A_{1}+F\right)$, $\left(\int+A_{1}+F\right) \partial=0$ since $\int \partial=1-e_{00} \in A_{1}+F$. Then the splitness implies that $\left(\int+f+A_{1}\right) \partial=0$ in $\mathbb{I}_{1} / A_{1}$ for some element $f \in F$, equivalently, $-e_{00}+f \partial \in A_{1} \cap F=0$ in $\mathbb{I}_{1}$, i.e. $f \partial=e_{00}$, this is obviously impossible (since $e_{i, j} \partial=e_{i, j+1}$ ), a contradiction.

Let $M$ be a module over a ring $R$. The socle $\operatorname{soc}_{R}(M)$, if nonzero, is the largest semi-simple submodule of $M$. The socle of $M$, if nonzero, is the only essential semi-simple submodule. The socle chain of the module $M$ is the ascending chain of its submodules:

$$
\operatorname{soc}_{R}^{0}(M):=\operatorname{soc}_{R}(M) \subseteq \operatorname{soc}_{R}^{1}(M) \subseteq \cdots \subseteq \operatorname{soc}_{R}^{i}(M) \subseteq \cdots
$$

where $\operatorname{soc}_{R}^{i}(M):=\varphi_{i-1}^{-1}\left(\operatorname{soc}_{R}\left(M / \operatorname{soc}_{R}^{i-1}(M)\right)\right)$ where $\varphi_{i-1}: M \rightarrow M / \operatorname{soc}^{i-1}(M), m \mapsto m+$ $\operatorname{soc}_{R}^{i-1}(M)$. Let $\operatorname{soc}_{R}^{\infty}(M):=\bigcup_{i \geq 0} \operatorname{soc}_{R}^{i}(M)$. If $M=\operatorname{soc}_{R}^{\infty}(M)$ then

$$
\operatorname{l.soc}_{R}(M)=1+\min \left\{i \geq 0 \mid M=\operatorname{soc}_{R}^{i}(M)\right\}
$$

is called the socle length of the $R$-module $M$. So, a nonzero module is semi-simple iff its socle length is 1 .

Theorem 2.5 1. The $A_{n}$-bimodule $\mathbb{I}_{n}$ is a holonomic $A_{2 n}$-module of length $3^{n}$ with pairwise non-isomorphic simple factors and each of them is the tensor product $\bigotimes_{i=1}^{n} M_{i}$ of simple $A_{2}(i)$-modules $M_{i}$ as in Theorem 2.3 for $i=1, \ldots, n$. Each simple factor $\bigotimes_{i=1}^{n} M_{i}$ is a simple holonomic $A_{2 n}$-module and has multiplicity 1 (with respect to the filtration of Bernstein on the algebra $A_{2 n}$ ) and its $A_{2 n}$-module endomorphism algebra is $K$.

2. $\operatorname{soc}_{A_{2 n}}\left(\mathbb{I}_{n}\right)=\bigotimes_{i=1}^{n} \operatorname{soc}_{A_{2}(i)}\left(\mathbb{I}_{1}(i)\right)=\bigotimes_{i=1}^{n}\left(A_{1}(i) \bigoplus F(i)\right)$.

3. The socle length of the $A_{2 n}$-module $\mathbb{I}_{n}$ is $n+1$. For each number $m=0,1, \ldots, n$,

$$
\operatorname{soc}_{A_{2 n}}^{m}\left(\mathbb{I}_{n}\right)=\sum_{i_{1}+\cdots+i_{n}=m} \bigotimes_{s=1}^{n} \operatorname{soc}_{A_{2}(i)}^{i_{s}}\left(\mathbb{I}_{1}(i)\right)
$$

where all $i_{s} \in\{0,1\}$ and $\operatorname{soc}_{A_{2}(i)}^{j}= \begin{cases}A_{1}(i) \oplus F(i) & \text { if } j=0, \\ \mathbb{I}_{1}(i) & \text { if } j=1 .\end{cases}$

4. For each number $m=0,1, \ldots, n$,

$$
\operatorname{soc}_{A_{2 n}}^{m}\left(\mathbb{I}_{n}\right) / \operatorname{soc}_{A_{2 n}}^{m-1}\left(\mathbb{I}_{n}\right)=\bigoplus_{i_{1}+\cdots+i_{n}=m} \bigotimes_{s=1}^{n} \operatorname{soc}_{A_{2}(i)}^{i_{s}}\left(\mathbb{I}_{1}(i)\right) / \operatorname{soc}_{A_{2}(i)}^{i_{s}-1}\left(\mathbb{I}_{1}(i)\right)
$$


and its length (as an $A_{2 n}$-module) is $\left(\begin{array}{c}n \\ m\end{array}\right) 2^{n-m}$ where all $i_{s} \in\{0,1\}$ and $\operatorname{soc}^{-1}:=0$.

5. The left $A_{2 n}$-module $\mathbb{I}_{n}$ has multiplicity $3^{n}$ with respect to the filtration of Bernstein of the Weyl algebra $A_{2 n}$.

Remark. The sum of lengths of all the factors in statement 4 is $3^{n}$ as

$$
3^{n}=(1+2)^{n}=\sum_{m=0}^{n}\left(\begin{array}{c}
n \\
m
\end{array}\right) 2^{n-m} .
$$

Proof. 1. By Theorem 2.3. (1), each of the tensor multiples $\mathbb{I}_{1}(i)$ in $\mathbb{I}_{n}=\bigotimes_{i=1}^{n} \mathbb{I}_{1}(i)$ has the $A_{2}(i)$-module (i.e. the $A_{1}(i)$-bimodule) filtration of length 3 with factors $M_{i}$ as in Theorem 2.3 (1). By considering the tensor product of these filtrations, the $A_{2 n}$-module $\mathbb{I}_{n}=\bigotimes_{i=1}^{n} \mathbb{I}_{1}(i)$ (i.e. the $A_{n}$-bimodule) has a filtration (of length $3^{n}$ ) with factors $\bigotimes_{i=1}^{n} M_{i}$. It is obvious that each $A_{2 n^{-}}$ module $\bigotimes_{i=1}^{n} M_{i}$ is isomorphic to a twisted $A_{2 n}$-module ${ }^{\sigma} P_{2 n}$ by an automorphism $\sigma$ of the Weyl algebra $A_{2 n}$ that preserves the filtration of Bernstein on the algebra $A_{2 n}$ where

$$
P_{2 n}=K\left[x_{1}, \ldots, x_{2 n}\right] \simeq A_{2 n} / \sum_{i=1}^{2 n} A_{2 n} \partial_{i} .
$$

This statement is obvious for $n=1$, then the general case follows at once. Since the $A_{2 n}$-module $P_{2 n}$ is simple, holonomic with multiplicity $1\left(\right.$ since $\left.e\left({ }^{\sigma} P_{2 n}\right)=e\left(P_{2 n}\right)=1\right)$ and $\operatorname{End}_{A_{2 n}}\left(P_{2 n}\right) \simeq K$, then so are all the $A_{2 n}$-modules $\bigotimes_{i=1}^{n} M_{i}$. This finishes the proof of statement 1 .

2. Statement 2 follows from statement 3 .

3. To prove statement 3 we use induction on $n$. The initial step when $n=1$ is true due to Theorem 2.3. (1). Suppose that $n>1$ and the statement holds for all $n^{\prime}<n$. Let $\left\{s^{0}=\right.$ $\left.A_{1} \oplus F, s^{1}=\mathbb{I}_{1}\right\}$ be the socle filtration for ${ }_{A_{1}} \mathbb{I}_{1 A_{1}}$ and let $\left\{s^{0}, s^{1}, \ldots, s^{n-1}\right\}$ be the socle filtration for $A_{n-1} \mathbb{I}_{n-1} A_{n-1}$. We are going to prove that

$$
\left\{s^{\prime 0}:=s^{0} \otimes s^{0}, s^{\prime 1}:=s^{0} \otimes s^{1}+s^{1} \otimes s^{0}, \ldots, s^{\prime n-1}:=s^{0} \otimes s^{n-1}+s^{1} \otimes s^{n-2}, s^{\prime n}:=s^{1} \otimes s^{n-1}\right\}
$$

is the socle filtration for $A_{n} \mathbb{I}_{n A_{n}}$. Notice that $A_{n}=A_{1} \otimes A_{n-1}, \mathbb{I}_{n}=\mathbb{I}_{1} \otimes \mathbb{I}_{n-1}$ and $\left\{s^{0} \otimes s^{i}\right\}_{i=0}^{n-1}$ is the socle filtration for $A_{n-1}\left(s^{0} \otimes \mathbb{I}_{n-1}\right)_{A_{n-1}}=s^{0} \otimes\left(A_{n-1} \mathbb{I}_{n-1} A_{n-1}\right)$ since the $\mathbb{I}_{n-1}$-bimodules $s^{0} \otimes s^{i} / s^{i-1}$ are semi-simple. Since, for each number $m=0,1, \ldots, n$, the $A_{n}$-subbimodule

$$
\bar{s}^{\prime m}:=s^{\prime m} / s^{\prime m-1}=s^{0} \otimes\left(s^{m} / s^{m-1}\right) \bigoplus\left(s^{1} / s^{0}\right) \otimes\left(s^{m-1} / s^{m-2}\right) \quad\left(\text { where } \bar{s}^{0}=s^{0} \otimes s^{0}\right)
$$

of $\mathbb{I}_{n}^{\prime} / s^{\prime m-1}$ is semi-simple, in order to finish the proof of statement 3 it suffices to show that $\bar{s}^{\prime m}$ is an essential $A_{n}$-subbimodule of $\mathbb{I}_{n} / s^{\prime m-1}$. Let $a$ be a nonzero element of the $A_{n}$-bimodule $\mathbb{I}_{n} / s^{\prime m-1}$. We have to show that $A_{n} a A_{n} \cap \bar{s}^{\prime m} \neq 0$. If $a \in s^{0} \otimes \mathbb{I}_{n-1}+s^{\prime m-1}$ then

$$
0 \neq \mathbb{I}_{n-1} a \mathbb{I}_{n-1} \cap s^{0} \otimes\left(s^{m} / s^{m-1}\right) \subseteq \bar{s}^{\prime m}
$$

(since $\left\{s^{0} \otimes s^{i}\right\}_{i=0}^{n-1}$ is the socle filtration for $\left.A_{n-1}\left(s^{0} \otimes \mathbb{I}_{n-1}\right)_{A_{n-1}}\right)$. If $a \notin s^{0} \otimes \mathbb{I}_{n-1}+s^{\prime m-1}$ then using the explicit basis $\left\{x_{1}^{i} x_{2}^{j}\right\}_{i, j \geq 0}$ for the $A_{1}$-bimodule $s^{1} / s^{0}$ (Proposition 2.2 (1)) and the action of the element $\partial$ on it (see (14)), we can find natural numbers, say $k$ and $l$, such that, by (13), the element

$$
a^{\prime}:=\partial^{k} a \partial^{l}=\int \otimes u_{1}+v_{2} \otimes u_{2}+\cdots+v_{s} \otimes u_{s},
$$

such that $0 \neq u_{1} \in \mathbb{I}_{n-1} / s^{m-1}$ (in particular, $a^{\prime}$ is a nonzero element of $\mathbb{I}_{n} / s^{\prime m-1}$ ); $u_{2}, \ldots, u_{s}$ are linearly independent elements of $\mathbb{I}_{n-1} ; v_{2}, \ldots, v_{s}$ are linearly independent elements of $s^{0}$. If the elements $u_{1}, u_{2}, \ldots, u_{s}$ are linearly independent then

$$
a^{\prime \prime}:=\partial a^{\prime}=1 \otimes u_{1}+\left(\partial v_{2}\right) \otimes u_{2}+\cdots+\left(\partial v_{s}\right) \otimes u_{s}
$$


is a nonzero element of $s^{0} \otimes \mathbb{I}_{n-1}$, and so, by the previous case $\mathbb{I}_{n} a \mathbb{I}_{n} \cap \bar{s}^{\prime m} \neq 0$.

If the elements $u_{1}, u_{2}, \ldots, u_{s}$ are linearly dependent then $u_{1}=\sum_{i=2}^{s} \lambda_{i} u_{i}$ for some elements $\lambda_{i} \in K$ not all of which are zero ones, say $\lambda_{2} \neq 0$. The element $a^{\prime}$ can be written as $a^{\prime}=$ $\left(\lambda_{2} \int+v_{2}\right) \otimes u_{2}+\cdots+\left(\lambda_{s} \int+v_{s}\right) \otimes u_{s}$.

$$
a^{\prime \prime}:=\partial a^{\prime}=\left(\lambda_{2}+\partial v_{2}\right) \otimes u_{2}+\cdots+\left(\lambda_{s}+\partial v_{s}\right) \otimes u_{s} .
$$

We claim that $a^{\prime \prime} \neq 0$. Suppose that $a^{\prime \prime}=0$, we seek a contradiction. Then $\lambda_{2}+\partial v_{2}=0, \ldots, \lambda_{s}+$ $\partial v_{s}=0$ in $A_{1} \oplus F$ (since the elements $u_{2}, \ldots, u_{s}$ are linearly independent). The first equality yields $0 \neq \lambda_{2}=\partial b$ in the Weyl algebra $A_{1}$ for some element $b \in A_{1}$. This is clearly impossible. Therefore, $a^{\prime \prime}$ is a nonzero element of $s^{0} \otimes \mathbb{I}_{n-1}$, and so, by the previous case, $\mathbb{I}_{n} a \mathbb{I}_{n} \cap \bar{s}^{\prime m} \neq 0$.

4. The equality follows from statement 3 . To prove the claim about the length note that $\left(\begin{array}{l}n \\ m\end{array}\right)$ is the number of vectors $\left(i_{1}, \ldots, i_{n}\right) \in\{0,1\}^{n}$ with $i_{1}+\cdots+i_{n}=m$; and for each choice of $\left(i_{1}, \ldots, i_{n}\right)$ the length of the $A_{2 n}$-module $\bigotimes_{s=1}^{n} \operatorname{soc}_{A_{2}(i)}^{i_{s}}\left(\mathbb{I}_{1}(i)\right) / \operatorname{soc}_{A_{2}(i)}^{i_{s}-1}\left(\mathbb{I}_{1}(i)\right)$ is $2^{n-m}$. Therefore, the length of the $A_{2 n}$-module $\operatorname{soc}_{A_{2 n}}^{m}\left(\mathbb{I}_{n}\right) / \operatorname{soc}_{A_{2 n}}^{m-1}\left(\mathbb{I}_{n}\right)$ is $\left(\begin{array}{l}n \\ m\end{array}\right) 2^{n-m}$.

5. Statement 5 follows from statement 1 and the additivity of the multiplicity on the holonomic modules.

\section{The algebra $\mathbb{I}_{n}$ is coherent iff $n=1$}

The aim of this section is to prove Theorem 3.1

A module $M$ over a ring $R$ is finitely presented if there is an exact sequence of modules $R^{m} \rightarrow R^{n} \rightarrow M \rightarrow 0$. A finitely generated module is a coherent module if every finitely generated submodule is finitely presented. A ring $R$ is a left (resp. right) coherent ring if the module ${ }_{R} R$ (resp. $R_{R}$ ) is coherent. A ring $R$ is a left coherent ring iff, for each element $r \in R, \operatorname{ker}_{R}(\cdot r)$ is a finitely generated left $R$-module and the intersection of two finitely generated left ideals is finitely generated, Proposition 13.3, [6]. Each left Noetherian ring is left coherent but not vice versa.

Theorem 3.1 The algebra $\mathbb{I}_{n}$ is a left coherent algebra iff the algebra $\mathbb{I}_{n}$ is a right coherent algebra iff $n=1$.

Proof. The first 'iff' is obvious since the algebra $\mathbb{I}_{n}$ is self-dual [1], i.e. is isomorphic to its opposite algebra $\mathbb{I}_{n}^{o p}$. If $n=1$ the algebra is a left coherent algebra 2. If $n \geq 2$ then the algebra $\mathbb{I}_{2}$ is not a left coherent algebra since, by Lemma 3.2 $\operatorname{ker}_{\mathbb{I}_{n}}\left(\cdot\left(H_{1}-H_{2}\right)\right)=\operatorname{ker}_{\mathbb{I}_{2}}\left(\cdot\left(H_{1}-H_{2}\right)\right) \otimes \mathbb{I}_{n-2} \simeq$ $\mathbb{I}_{n}\left(P_{2} \otimes \mathbb{I}_{n-2}\right)^{(\mathbb{N})}$ is an infinite direct sum of nonzero $\mathbb{I}_{n}$-modules, hence it is not finitely generated. Therefore, the algebra $\mathbb{I}_{n}$ is not a left coherent algebra, by Proposition 13.3, 6].

Lemma $3.2 \operatorname{ker}_{\mathbb{I}_{2}}\left(\cdot\left(H_{1}-H_{2}\right)\right)=\operatorname{ker}_{F_{2}}\left(\cdot\left(H_{1}-H_{2}\right)\right)=\bigoplus_{i, j, k \in \mathbb{N}} K e_{i j}(1) e_{k j}(2) \simeq\left(\mathbb{I}_{2} P_{2}\right)^{(\mathbb{N})}$.

Proof. The algebra $B_{2}=\mathbb{I}_{2} / \mathfrak{a}_{2}$ is a domain 1] where $\mathfrak{a}_{2}:=F(1) \otimes \mathbb{I}_{1}(2)+\mathbb{I}_{1}(1) \otimes F(2)$ and $H_{1}-H_{2} \notin \mathfrak{a}_{2}$. Therefore, $\mathcal{K}:=\operatorname{ker}_{\mathbb{I}_{2}}\left(\cdot\left(H_{1}-H_{2}\right)\right)=\operatorname{ker}_{\mathfrak{a}_{2}}\left(\cdot\left(H_{1}-H_{2}\right)\right)$. Let $F_{2}:=F(1) \otimes F(2)$. Notice that

$$
\mathbb{I}_{2}\left(\mathfrak{a}_{2} / F_{2}\right)_{\mathbb{I}_{2}} \simeq F(1) \otimes B_{1}(2) \bigoplus B_{1}(1) \otimes F(2)
$$

is a direct sum of two $\mathbb{I}_{2}$-bimodules. It follows from the presentation $F(1) \otimes B_{1}(2)=\bigoplus_{i, j \in \mathbb{N}, k \in \mathbb{Z}} e_{i j}(1) \otimes$ $\partial_{2}^{k} K\left[H_{2}\right]$ that $\operatorname{ker}_{F(1) \otimes B_{1}(2)}\left(\cdot\left(H_{1}-H_{2}\right)\right)=0$. Similarly, $\operatorname{ker}_{B_{1}(1) \otimes F(2)}\left(\cdot\left(H_{1}-H_{2}\right)\right)=0$ (or use the $(1,2)$-symmetry). Therefore,

$$
\mathcal{K}=\operatorname{ker}_{F_{2}}\left(\cdot\left(H_{1}-H_{2}\right)\right)=\bigoplus_{i, j, k \in \mathbb{N}} K e_{i j}(1) e_{k j}(2)=\bigoplus_{j \in \mathbb{N}}\left(\bigoplus_{i, k \in \mathbb{N}} K e_{i j}(1) e_{k j}(2)\right) \simeq \bigoplus_{j \in \mathbb{N}}\left(\mathbb{I}_{2} P_{2}\right) \simeq \mathbb{I}_{2}\left(P_{2}\right)^{(\mathbb{N})}
$$




\section{The algebra $\mathbb{I}_{n}$ is maximal order}

The aim of this section is to prove Theorem 4.3 ,

Let $R$ be a ring. An element $x \in R$ is right regular if $x r=0$ implies $r=0$ for $r \in R$. Similarly, a left regular element is defined. A left and right regular element is called a regular element. The sets of regular/left regular/right regular elements of a ring $R$ are denoted respectively by $\mathcal{C}_{R}(0),{ }^{\prime} \mathcal{C}_{R}(0)$ and $\mathcal{C}_{R}^{\prime}(0)$. For an arbitrary ring $R$ there exists the largest (w.r.t. inclusion) left regular denominator set $S_{l, 0}=S_{l, 0}(R)$ in the ring $R$ (regular means that $S_{l, 0}(R) \subseteq \mathcal{C}_{R}(0)$ ), and so $Q_{l}(R):=S_{l, 0}^{-1} R$ is the largest left quotient ring of $R$ (Theorem 2.1, 4 4 ). Similarly, for an arbitrary ring $R$ there exists the largest right regular denominator set $S_{r, 0}=S_{r, 0}(R)$ in $R$, and so $Q_{r}(R):=R S_{r, 0}^{-1}$ is the largest right quotient ring of $R$. The rings $Q_{l}(R)$ and $Q_{r}(R)$ were introduced and studied in [4. Let $\operatorname{End}_{K}(K[x])$ be the algebra of all linear maps from the vector space $K[x]$ to itself and $\operatorname{Aut}_{K}(K[x])$ be its group of units (i.e. the group of all invertible linear maps from $K[x]$ to itself). The algebra $\mathbb{I}_{1}$ is a subalgebra of $\operatorname{End}_{K}(K[x])$. Theorem 5.6.(1), [4, states that $S_{r, 0}\left(\mathbb{I}_{1}\right)=\mathbb{I}_{1} \cap \operatorname{Aut}_{K}(K[x])$, it is the set of all elements of the algebra $\mathbb{I}_{1}$ that are invertible linear maps in $K[x]$. The set $S_{r, 0}\left(\mathbb{I}_{1}\right)$ is huge comparing to the group of units $\mathbb{I}_{1}^{*}$ of the algebra $\mathbb{I}_{1}$ which is obviously a subset of $S_{r, 0}\left(\mathbb{I}_{1}\right)$.

Let $R$ be a ring. A subring $S$ (not necessarily with 1 ) of the largest right quotient ring $Q_{r}(R)$ of the ring $R$ is called a right order in $Q_{r}(R)$ if each element $q \in Q_{r}(R)$ has the form $r s^{-1}$ for some elements $r, s \in S$. A subring $S$ (not necessarily with 1 ) of the largest left quotient ring $Q_{l}(R)$ of the ring $R$ is called a left order in $Q_{l}(R)$ if each element $q \in Q_{l}(R)$ has the form $s^{-1} r$ for some elements $r, s \in S$.

Let $R_{1}$ and $R_{2}$ be right orders in $Q_{r}\left(\mathbb{I}_{n}\right)$. We say that the right orders $R_{1}$ and $R_{2}$ are equivalent, $R_{1} \sim R_{2}$, if there are units $a_{1}, a_{2}, b_{1}, b_{2} \in Q_{r}\left(\mathbb{I}_{n}\right)$ such that $a_{1} R_{1} b_{1} \subseteq R_{2}$ and $a_{2} R_{2} b_{2} \subseteq R_{1}$. Clearly, $\sim$ is an equivalent relation on the set of right orders in $Q_{r}\left(\mathbb{I}_{n}\right)$. A right order in $Q_{r}\left(\mathbb{I}_{n}\right)$ is called a maximal right order if it is maximal (w.r.t. $\subseteq$ ) within its equivalence class.

Lemma 4.1 Let $Q_{r}\left(\mathbb{I}_{n}\right)$ be the right quotient ring of $\mathbb{I}_{n}$ and $R, S$ be equivalent right orders in $Q_{r}\left(\mathbb{I}_{n}\right)$ such that $R \subseteq S$. Then there are equivalent right orders $T$ and $T^{\prime}$ in $Q_{r}\left(\mathbb{I}_{n}\right)$ with $R \subseteq$ $T \subseteq S, R \subseteq T^{\prime} \subseteq S$ and units $r_{1}, r_{2}$ of $Q_{r}\left(\mathbb{I}_{n}\right)$ contained in $R$ such that $r_{1} S \subseteq T, T r_{2} \subseteq R$ and $S r_{2} \subseteq T^{\prime}, r_{1} T^{\prime} \subseteq R$. In particular, $r_{1} S r_{2} \subseteq R$.

Proof. By definition, $a S b \subseteq R$ for some units $a, b$ of $Q_{r}\left(\mathbb{I}_{n}\right)$. Then $a=r_{1} s_{1}^{-1}$ and $b=r_{2} s_{2}^{-1}$ with $r_{i}, s_{i} \in R$. Then $r_{1} S r_{2} \subseteq r_{1} s_{1}^{-1} S r_{2} \subseteq R s_{2} \subseteq R$. It is readily checked that $T=R+r_{1} S+R r_{1} S$ and $T^{\prime}=R+S r_{2}+S r_{2} R$ are as claimed.

Lemma $4.2 \quad$ 1. $\mathcal{C}_{\mathbb{I}_{n}}(0) \cap \mathfrak{a}_{n}=\emptyset,{ }^{\prime} \mathcal{C}_{\mathbb{I}_{n}}(0) \cap \mathfrak{a}_{n}=\emptyset$ and $\mathcal{C}_{\mathbb{I}_{n}}^{\prime}(0) \cap \mathfrak{a}_{n}=\emptyset$.

2. $S_{l, 0}\left(\mathbb{I}_{n}\right) \cap \mathfrak{a}_{n}=\emptyset$ and $S_{r, 0}\left(\mathbb{I}_{n}\right) \cap \mathfrak{a}_{n}=\emptyset$.

3. For all elements $a \in S_{l, 0}\left(\mathbb{I}_{n}\right) \cup S_{r, 0}\left(\mathbb{I}_{n}\right), \mathbb{I}_{n} a \mathbb{I}_{n}=\mathbb{I}_{n}$.

Proof. 1. Trivial (since every element of the ideal $\mathfrak{a}_{n}$ is a left and right zero divisor in $\mathbb{I}_{n}$ ).

2. Statement 2 follows from statement 1 and the inclusions $S_{l, 0}\left(\mathbb{I}_{n}\right), S_{r, 0}\left(\mathbb{I}_{n}\right) \subseteq \mathcal{C}_{\mathbb{I}_{n}}(0)$.

3. If $\mathbb{I}_{n} a \mathbb{I}_{n} \neq \mathbb{I}_{n}$ for some element $a \in S_{l, 0}\left(\mathbb{I}_{n}\right) \cup S_{r, 0}\left(\mathbb{I}_{n}\right)$ then $a \in \mathbb{I}_{n} a \mathbb{I}_{n} \subseteq \mathfrak{a}_{n}$ (as $\mathfrak{a}_{n}$ is the only maximal ideal of the algebra $\mathbb{I}_{n}$ ). This contradicts to statement 2 .

Theorem 4.3 The algebra $\mathbb{I}_{n}$ is a maximal left order in $Q_{l}\left(\mathbb{I}_{n}\right)$ and a maximal right order in $Q_{r}\left(\mathbb{I}_{n}\right)$.

Proof. Suppose that $\mathbb{I}_{n} \subseteq S$ and $S \sim \mathbb{I}_{n}$ for some right order $S$ in $Q_{r}\left(\mathbb{I}_{n}\right)$. Then $a S b \subseteq \mathbb{I}_{n}$ for some elements $a, b \in \mathbb{I}_{n} \cap Q_{r}\left(\mathbb{I}_{n}\right)^{*}$, by Lemma 4.1, where $Q_{r}\left(\mathbb{I}_{n}\right)^{*}$ is the group of units of the algebra $Q_{r}\left(\mathbb{I}_{n}\right)$. By Theorem 2.8, 4, $\mathbb{I}_{n} \cap Q_{r}\left(\mathbb{I}_{n}\right)^{*}=S_{r, 0}\left(\mathbb{I}_{n}\right)$. Then, by Corollary 4.2. (3), $\mathbb{I}_{n} \supseteq \mathbb{I}_{n} a S b \mathbb{I}_{n}=\left(\mathbb{I}_{n} a \mathbb{I}_{n}\right) S\left(\mathbb{I}_{n} b \mathbb{I}_{n}\right)=\mathbb{I}_{n} S \mathbb{I}_{n}=S$, i.e. $\mathbb{I}_{n}=S$. Then the algebra $\mathbb{I}_{n}$ is a maximal right order in $Q_{r}\left(\mathbb{I}_{n}\right)$. Since the algebra $\mathbb{I}_{n}$ admits an involution [1, the algebra $\mathbb{I}_{n}$ is also a maximal left order in $Q_{l}\left(\mathbb{I}_{n}\right)$. 


\section{References}

[1] V. V. Bavula, The algebra of integro-differential operators on a polynomial algebra, Journal of the London Math. Soc. DOI:10.1112/jlms/jdq081, (Arxiv:math.RA: 0912.0723).

[2] V. V. Bavula, The algebra of integro-differential operators on an affine line and its modules, (Arxiv:math.RA: 1011.2997).

[3] V. V. Bavula, An analogue of the Conjecture of Dixmier is true for the algebra of polynomial integrodifferential operators, (Arxiv:math.RA: 1011.3009).

[4] V. V. Bavula, The largest left quotient ring of a ring, (Arxiv:math.RA:1101.5107).

[5] I. N. Bernstein The analitic continuation of generalized functions with respect to a parameter, Funct. Anal. and Appl. 6 (1972), no. 4, 26-40.

[6] B. Stenström, Rings of quotients. An introduction to methods of ring theory. Springer-Verlag, New York-Heidelberg, 1975.

Department of Pure Mathematics

University of Sheffield

Hicks Building

Sheffield S3 7RH

UK

email: v.bavula@sheffield.ac.uk 